\title{
Triterpenoid Saponins from Astragalus corniculatus
}

\author{
Ilina Krasteva ${ }^{\mathrm{a}}$, Stefan Nikolov ${ }^{\mathrm{a}}$, Maki Kaloga ${ }^{\mathrm{b}}$, and Gisela Mayer ${ }^{\mathrm{c}}$ \\ ${ }^{a}$ Department of Pharmacognosy, Faculty of Pharmacy, 2 Dunav St., 1000 Sofia, Bulgaria \\ ${ }^{\mathrm{b}}$ Institut für Pharmazie, Pharmazeutische Biologie, Königin Luise Straße 2+4, D-14195 Berlin, \\ Germany \\ ${ }^{c}$ Schering AG, Abt. IPCH Strukturanalyse, D-13353, Berlin, Germany \\ Reprint requests to Dr. I. Krasteva. Fax: (+359 2) 9879874. E-mail: ikrasteva@pharmfac.acad.bg
}

Z. Naturforsch. 61b, 1166 - 1169 (2006); received April 24, 2006

Two new triterpenoid saponins were isolated from the ethanolic extract of the aerial parts of Astragalus corniculatus Bieb. The structures of the saponins were elucidated as $3 \beta$-O-[O-4-oxopentopyranosyl-( $1 \rightarrow 2)$ - $\beta$-D-glucopyranosyl]-21 $\alpha$-hydroxyolean-12-ene-28-oic acid (1) and $21 \alpha$ hydroxyolean-12-ene-28-oic acid 3 $\beta$-4-oxo-pentopyranoside (2) by chemical and spectral methods.

Key words: Astragalus corniculatus, Fabaceae, Triterpenes

\section{Introduction}

Astragalus corniculatus Bieb. (Fabaceae) is grown in North Bulgaria and distributed in Southeastern Romania, South Ukraine and Moldova. Many species of Astragalus L. have been recorded as yielding a wide range of saponins. Although tetracyclic triterpene saponins are widely distributed in genus Astragalus, pentacyclic triterpenes do not often occur [1-4]. In a previous work we reported on a protective effect of purified mixture of saponins from A. corniculatus Bieb. against myeloid Graffi tumors [5]. However, no work has been reported on the saponins of this species.

The present paper describes the isolation and identification of two new oleanane type saponins (1 and 2) from A. corniculatus.

\section{Results and Discussion}

Solvent partition and repeated chromatographic purification over silica gel and Sephadex LH-20 of the saponin mixture obtained from ethanolic extract of the aerial parts of Astragalus corniculatus afforded two new triterpenoid saponins, $\mathbf{1}$ and 2. Sapogenin 1a was obtained after acid hydrolysis of a saponin mixture, followed by column chromatography on Sephadex LH-20 and preparative TLC. Acid and enzymatic hydrolysis of $\mathbf{1}$ and $\mathbf{2}$ afforded the same genin 1a, Dglucose and 4-oxo-pentose.

The negative ion FAB-MS spectrum of compound 1 showed the quasi-molecular ion $[\mathrm{M}-\mathrm{H}]^{-}$ peak at $m / z=763$ together with sodium adducts at $m / z=785[(\mathrm{M}-\mathrm{H}+\mathrm{Na})-\mathrm{H}]^{-}$and $m / z=807$ $[(\mathrm{M}-2 \mathrm{H}+2 \mathrm{Na})-\mathrm{H}]^{-}$, and two other important ion peaks appeared at $m / z=633$ [(M-H)-130] $^{-}$and $m / z=471$ [(M-H)-130-162 $]^{-}$indicating the respective elimination of 130 mass units and a glucosyl moiety. The positive ion FAB-MS of 1 exhibited a $[(\mathrm{M}-2 \mathrm{H}+2 \mathrm{Na})+\mathrm{H}]^{+}$peak at $m / z=809$. Its HRESI MS spectrum showed the $[\mathrm{M}-\mathrm{H}]^{-}$ion peak at $m / z=763.4328$, corresponding to the molecular formula $\mathrm{C}_{41} \mathrm{H}_{64} \mathrm{O}_{13}$. The ${ }^{1} \mathrm{H}$ NMR spectrum of saponin $\mathbf{1}$ and the less polar compound $\mathbf{2}$ exhibited seven $\mathrm{Me}$ singlets and signals for an olefinic proton, and two oxymethine proton resonances at $\delta_{\mathrm{H}}=3.21$ (overlap.) and 3.42 (br t-like, $J=3.50 \mathrm{~Hz}$ ) for $\mathbf{1}$, and $\delta=3.20$ (dd, $J=11.70,4.30 \mathrm{~Hz}, \mathrm{H}-3 \alpha$ ) and 3.42 (overlap.) for 2 , respectively. The ${ }^{1} \mathrm{H}$ and ${ }^{13} \mathrm{C}$ NMR data of $\mathbf{1}$ clearly revealed the presence of two sugar moieties. The anomeric proton signals appeared at $\delta_{\mathrm{H}}=4.67(\mathrm{~d}$, $J=7.70 \mathrm{~Hz}, \mathrm{H}-1$ ') and 4.45 (d, $J=7.40 \mathrm{~Hz}, \mathrm{H}-1$ '), which showed 1,2 diaxial coupling. In the HMBC spectrum correlations were observed between these protons (H-1' and H-1") and C-3 and C-2', respectively. This identified the points of attachment of the sugar units as C-3 $\left(\delta_{\mathrm{C}}=91.46\right)$ and C-2' $(\delta=81.33)$.

The EIMS of sapogenin 1a showed a fragment at $m / z=454$ generated from the $[\mathrm{M}]^{+}$(absent in the spectrum). The presence of significant peaks at $m / z=246$ [RDA product] $^{+}$and $m / z=201$ [246$\mathrm{COOH}]^{+}$(base peak), suggested the presence of one hydroxyl group and a carboxyl group in the D/E rings. These fragments were recorded in the HR-EIMS at 
Table 1. NMR spectral data of $\mathbf{1 a}$ in $\mathrm{CD}_{3} \mathrm{OD}$ ( $\delta$ values).

\begin{tabular}{|c|c|c|c|}
\hline Position & ${ }^{13} \mathrm{C}$ & ${ }^{1} \mathrm{H}$ & HMBC \\
\hline$\overline{1}$ & 39.85 & $1.64 / 1.10$ & \\
\hline 2 & 28.20 & $\begin{array}{l}2.20, \mathrm{dt}, 13.80,3.00, \mathrm{H}-2 \mathrm{ax} \\
1.79, \mathrm{~m}, \mathrm{H}-2 \mathrm{eq}\end{array}$ & \\
\hline 3 & 79.84 & $3.14, \mathrm{dd}, 11.30,4.80, \mathrm{H}-3 \alpha$ & $\mathrm{H}_{3}-23, \mathrm{H}_{2}-24$ \\
\hline 4 & 39.96 & - & $\mathrm{H}_{3}-23, \mathrm{H}_{3}-24$ \\
\hline 5 & 56.93 & 0.75 , brd, $11.00, \mathrm{H}-5$ & $\mathrm{H}_{3}-23, \mathrm{H}_{3}-24, \mathrm{H}_{3}-25$ \\
\hline 6 & 19.58 & $1.55 / 1.40$ & \\
\hline 7 & 34.39 & $1.34, \mathrm{~m}, \mathrm{H}_{2}-7$ & $\mathrm{H}_{3}-26$ \\
\hline 8 & 40.20 & - & $\mathrm{H}_{3}-26, \mathrm{H}_{3}-27$ \\
\hline 9 & 49.32 & 1.58 & $\mathrm{H}-12, \mathrm{H}_{3}-25, \mathrm{H}_{3}-26$ \\
\hline 10 & 38.25 & - & \\
\hline 11 & 24.62 & $1.88, \mathrm{~m}, \mathrm{H}_{2}-11$ & \\
\hline 12 & 122.49 & 5.23, t-like, 3.40, H-12 & \\
\hline 13 & 146.63 & - & $\mathrm{H}_{2}-11, \mathrm{H}-18, \mathrm{H}_{3}-27$ \\
\hline 14 & 40.34 & - & $\mathrm{H}-18$ \\
\hline 15 & 29.60 & - & $\mathrm{H}_{3}-27$ \\
\hline 16 & 24.97 & $1.62 / 1.58$ & \\
\hline 17 & 49.00 & - & H-18, H-21 \\
\hline 18 & 43.16 & 2.99 , br dd, $13.70,3.40$ & H-12, H-19ax \\
\hline 19 & 43.66 & $\begin{array}{l}\text { 2.06, t-like, } 13.70, \mathrm{H}-19 \mathrm{ax} \\
0.97, \mathrm{~m}, \mathrm{H}-19 \mathrm{eq}, \\
\text { overlap. with } \mathrm{H}_{3}-29\end{array}$ & $\begin{array}{l}\mathrm{H}-18, \mathrm{H}-21, \mathrm{H}_{3}-29, \\
\mathrm{H}_{3}-30\end{array}$ \\
\hline 20 & 36.20 & - & $\begin{array}{l}\mathrm{H}-18, \mathrm{H}-19 \mathrm{ax}, \mathrm{H}-21, \\
\mathrm{H}_{3}-29, \mathrm{H}_{3}-30\end{array}$ \\
\hline 21 & 75.92 & 3.42 , br t-like, $3.50, \mathrm{H}-21 \beta$ & $\mathrm{H}_{3}-29, \mathrm{H}_{3}-30$ \\
\hline 22 & 41.25 & $1.90 / 1.70$ & \\
\hline 23 & 28.78 & $0.97, \mathrm{~s}, \mathrm{H}_{3}-23$ & $\mathrm{H}-3 \alpha, \mathrm{H}_{3}-24$ \\
\hline 24 & 16.33 & $0.77, \mathrm{~s}, \mathrm{H}_{3}-24$ & $\mathrm{H}-3 \alpha, \mathrm{H}_{3}-23$ \\
\hline 25 & 15.99 & $0.94, \mathrm{~s}, \mathrm{H}_{3}-25$ & \\
\hline 26 & 18.26 & $0.88, \mathrm{~s}, \mathrm{H}_{3}-26$ & \\
\hline 27 & 25.94 & $1.15, \mathrm{~s}, \mathrm{H}_{3}-27$ & \\
\hline 28 & 180.38 & - & $\mathrm{H}-22 \mathrm{~b}$ \\
\hline 29 & 27.96 & $0.98, \mathrm{~s}, \mathrm{H}_{3}-29$ & H-19ax, H-21, $\mathrm{H}_{3}-30$ \\
\hline 30 & 25.80 & $0.89, \mathrm{~s}, \mathrm{H}_{3}-30$ & \\
\hline
\end{tabular}

$m / z=454.34459$ (calcd. 454.34467 for $\mathrm{C}_{30} \mathrm{H}_{46} \mathrm{O}_{3}$ ), $m / z=246.16195$ (calcd. 246.16198 for $\mathrm{C}_{16} \mathrm{H}_{22} \mathrm{O}_{2}$ ) and $m / z=201.16422$ (calcd. 201.16433 for $\mathrm{C}_{15} \mathrm{H}_{21}$ ). In the desorption chemical ionisation (DCI) spectrum, 1a gave a molecular ion peak at $\mathrm{m} / z=472$ $\left[\mathrm{C}_{30} \mathrm{H}_{48} \mathrm{O}_{4}\right]$. The ${ }^{1} \mathrm{H}$ NMR spectrum of $\mathbf{1 a}$ displayed signals for seven tertiary methyl groups at $\delta_{\mathrm{H}}=0.77$, $0.88,0.89,0.94,0.97,0.98$, and 1.15 (each s), an olefinic proton at 5.23 (t-like, $J=3.40 \mathrm{~Hz}$ ), and oneproton signal due to $\mathrm{H}-18 \beta$, characteristic of olean12-ene-28-oic acid type triterpenes [6] was observed at $\delta=2.99(\mathrm{dd}, J=13.70,3.40)$. This ${ }^{1} \mathrm{H}$ NMR spectrum indicated also the existence of two hydroxylated methine signals at $\delta_{\mathrm{H}}=3.14(\mathrm{dd}, J=11.30,4.80 \mathrm{~Hz}$, $\mathrm{H}-3 \alpha$ ) and $\delta=3.42$ (br t-like, $J=3.50 \mathrm{~Hz}$ ). The position of the second $\mathrm{OH}$ group was determined to be $\mathrm{C}-21$ by $\mathrm{HMBC}$ correlations of $\mathrm{H}-21$ with $\mathrm{C}-17$ $\left(\delta_{\mathrm{C}}=49.00\right), \mathrm{C}-19(\delta=43.66), \mathrm{C}-20(\delta=36.20)$, C-29 $(\delta=27.96)$, and C-30 $(\delta=25.80)$ (Table 1$)$.

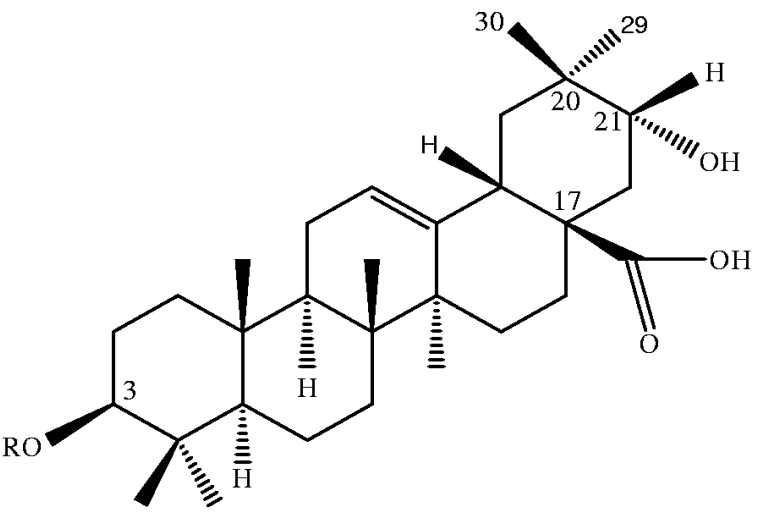

1a: $\mathrm{R}=\mathrm{H}$;

$1: \mathrm{R}=4$-oxo-pentopyranosyl- $(1 \rightarrow 2)-\beta$-D-glcp;

$2: \mathrm{R}=4$-oxo-pentopyranosyl.

Further the proton signal H-21 at $\delta=3.42$ was observed as broad triple t-like signal $(J=3.50 \mathrm{~Hz})$. This confirmed that the hydroxyl group on ring $\mathrm{E}$ of $1 \mathrm{a}$ must be $\alpha$-located [7]. The NOE associations in the NOESY spectrum between $\mathrm{H}-21 / \mathrm{H}_{3}-29$ and $\mathrm{H}_{3}-30$ supported the $\alpha$-configuration of the 21-hydroxy group.

The structure of 1a was therefore determined as $3 \beta, 21 \alpha$-dihydroxyolean-12-ene-28-oic acid and $\mathbf{1}$ as $3 \beta$-O-[O-4-oxo-pentopyranosyl- $(1 \rightarrow 2)-\beta$-D-glucopyranosyl]-21 $\alpha$-hydroxyolean-12-ene-28-oic acid.

Compound 2 showed the quasi-molecular ion [M$\mathrm{H}]^{-}$at $m / z=601$ and a prominent fragment ion peak at $m / z=471[(\mathrm{M}-\mathrm{H})-130]^{-}$in the negative ion FAB-MS spectrum. The positive FAB-MS revealed an $[(\mathrm{M}-2 \mathrm{H}+2 \mathrm{Na})+\mathrm{H}]^{+}$ion at $m / z=647$. Its HR-ESI MS spectrum gave the $[\mathrm{M}-\mathrm{H}]^{-}$ion peak at $m / z=$ 601.3798 , indicating the molecular formula $\mathrm{C}_{35} \mathrm{H}_{54} \mathrm{O}_{8}$. The ${ }^{13} \mathrm{C}$ NMR spectrum of $\mathbf{2}$ was similar to that of $\mathbf{1}$ but featured one less glucosyl moiety, and displayed a signal characteristic of an anomeric carbon at $\delta_{\mathrm{C}}=106.72$ (C-1'). The glycosidic connectivity in 2 was further confirmed by correlation between anomeric proton $(\delta=4.34, \mathrm{~d}, J=7.70 \mathrm{~Hz})$ and C-3 $(\delta=90.96)$ of the aglycone. On the other hand, the large coupling constant $(J=7.70 \mathrm{~Hz})$ observed for this anomeric proton indicated it to be axially oriented.

Thus, we conclude that saponin 2 is $21 \alpha$-hydroxyolean-12-ene-28-oic acid 3 $\beta$-4-oxo-pentopyranoside.

Structure determination of these compounds was established on the basis of spectral data. The ${ }^{13} \mathrm{C}$ and ${ }^{1} \mathrm{H}$ NMR spectra of $\mathbf{1}, \mathbf{1 a}$ and $\mathbf{2}$ have been assigned using ${ }^{13} \mathrm{C}$ DEPT, ${ }^{1} \mathrm{H},{ }^{1} \mathrm{H}$ COSY, HETCOR, HMBC and NOESY techniques. 


\section{Experimental Section}

General

Melting points are uncorrected. Optical rotations were measured with a Perkin-Elmer 343 polarimeter. IR spectra were obtained with a Shimadzu FTIR-8101 M spectrometer. ${ }^{1} \mathrm{H}$ NMR (400 MHz) and ${ }^{13} \mathrm{C}$ NMR (100.6 MHz) spectra were recorded on Bruker DPX - 400 and Bruker AMX 400 instruments using TMS as internal standard. All spectra were recorded in $\mathrm{CD}_{3} \mathrm{OD}$. $\mathrm{HMBC}$ experiments were optimised for ${ }^{2-3} J_{\mathrm{H} / \mathrm{C}}=8 \mathrm{~Hz}$. EIMS, HR-EIMS, FAB-MS and $\mathrm{DCI} / \mathrm{NH}_{3}$ spectra were carried out Varian $\mathrm{MAT} \mathrm{CH}_{7} \mathrm{~A}$, Finnigan MAT 711, Finnigan MAT $\mathrm{CH}_{5} \mathrm{DF}$ and Finnigan TSQ 700 spectrometers, respectively. HR-ESI MS spectra were recorded on a Q-Tof Dremier mass spectrometer (Firma Waters). Thin-layer chromatographic study (TLC) was carried out on Kieselgel $60 \mathrm{~F}_{254}$ ( $0.24 \mathrm{~mm}$ thick, Merck) plates, using the solvent systems 1-BuOH-AcOH-H$-\mathrm{H}_{2} \mathrm{O}(4: 1: 1)$ and $\mathrm{CHCl}_{3}-\mathrm{MeOH}(25: 1)$. The spots were visualized by spraying with anisaldehyde/conc. $\mathrm{H}_{2} \mathrm{SO}_{4}$ (for saponins and sapogenins) and thymol/conc. $\mathrm{H}_{2} \mathrm{SO}_{4}$ solution (for sugars), followed by heating at $110{ }^{\circ} \mathrm{C}$. Column chromatography (CC) was carried out with Sephadex LH-20 and silica gel 60 (70230 mesh, Merck). Prep. TLC was performed on silica gel plates (Kieselgel 60, $0.5 \mathrm{~mm}$ thick, Merck).

\section{Plant material, extraction and isolation}

Astragalus corniculatus Bieb. herbs were collected in July 1999 in Northern Bulgaria. The plant was identified by Dr. D. Pavlova from the Department of Botany, Faculty of Biology, Sofia University, where voucher specimen has been deposited (SO95265). The air-dried plant material $(1 \mathrm{~kg})$ was powdered and extracted exhaustively with $50 \% \mathrm{EtOH}$. The extract was filtrated, concentrated under reduced pressure and successively treated with $\mathrm{CHCl}_{3}$ and EtOAc respectively. The residue was dissolved in $\mathrm{MeOH}$. After filtration and addition of $\mathrm{Me}_{2} \mathrm{CO}$ a precipitate (45 g) was obtained. $20 \mathrm{~g}$ of the precipitate were chromatographed on a silica gel column eluting with $\mathrm{CHCl}_{3}-\mathrm{MeOH}-\mathrm{H}_{2} \mathrm{O}$ (98: $72: 9$ ) to give five crude fractions (A-E). Fr. C was repeatedly chromatographed on Sephadex LH-20 eluting with $\mathrm{MeOH}$ and on silica gel $\left(\mathrm{CHCl}_{3}-\mathrm{MeOH}-\mathrm{H}_{2} \mathrm{O} 98: 72: 9\right)$ to yield a fraction, containing compounds $\mathbf{1}$ and 2. This fraction $(182 \mathrm{mg})$ was purified by prep. TLC on silica gel $\mathrm{G}$ developed in 1 - BuOH-AcOH- $\mathrm{H}_{2} \mathrm{O}(4: 1: 1)$ to give $1(23 \mathrm{mg})$ and $2(20 \mathrm{mg})$. Another $20 \mathrm{~g}$ of the precipitate were refluxed with $8 \% \mathrm{H}_{2} \mathrm{SO}_{4}$ for $6 \mathrm{~h}$ and separated by centrifugation and filtration. The obtained solution was neutralized and evaporated to give $6 \mathrm{~g}$ of a crude sapogenin mixture, which was separated into 10 fractions (each $150 \mathrm{ml}$ ) by CC on Sephadex LH-20 eluted with methanol to give three main fractions (I-III). Fr. II was rechromatographed on Sephadex LH-20 with $\mathrm{MeOH}$ and appropriate fractions were com- bined (IIa-IIc). Fraction IIb (129 mg) was subjected to prep. TLC with $\mathrm{CHCl}_{3}-\mathrm{MeOH}(25: 1)$ to afford a sapogenin 1a (15 mg).

\section{Isolated sapogenin and saponins}

$3 \beta, 21 \alpha$-dihydroxyolean-12-ene-28-oic acid (1a): Amorphous powder, m. p. $240-243{ }^{\circ} \mathrm{C} ;[\alpha]_{\mathrm{D}}^{20}=+15.3^{\circ}(\mathrm{MeOH}$, c 0.30). $-{ }^{1} \mathrm{H}$ and ${ }^{13} \mathrm{C}$ NMR: (Table 1). - MS (EI, $70 \mathrm{eV}$ ): $m / z$ (rel. int.) $454\left[\mathrm{M}-\mathrm{H}_{2} \mathrm{O}\right]^{+}, 246$ [RDA product], 201 (base peak); DCI/NH $\mathrm{NH}_{3}: m / z=472[\mathrm{M}]^{+}$; HR-EIMS $m / z=$ 454.34459 (calcd. 454.34467 for $\mathrm{C}_{30} \mathrm{H}_{46} \mathrm{O}_{3}$ ).

$3 \beta$-O-[O-4-oxo-pentopyranosyl- $(1 \rightarrow 2)-\beta$-D-glucopyranosyl]-21 $\alpha$-hydroxyolean-12-ene-28-oic acid (1): Amorphous powder, m. p. $191-192{ }^{\circ} \mathrm{C} ;[\alpha]_{\mathrm{D}}^{20}=+8^{\circ}(\mathrm{MeOH}$, $c$ 0.35). - IR (KBr) $v_{\max } 3421(\mathrm{OH}), 2929,1725(\mathrm{C}=\mathrm{O})$, $1618,1075,1044 \mathrm{~cm}^{-1} .-{ }^{1} \mathrm{H}$ NMR $\left(400 \mathrm{MHz}, \mathrm{CD}_{3} \mathrm{OD}\right)$ : $\delta=0.85,0.87,0.90,0.95,0.98,1.07,1.15(3 \mathrm{H}$ each, tertiary methyls), 0.77 (br d, $J=11.00 \mathrm{~Hz}$ ), 3.21 (overlap., $\mathrm{H}-3 \alpha$ ), 3.42 (br t-like, $J=3.50 \mathrm{~Hz}, \mathrm{H}-21 \beta), 4.67(\mathrm{~d}, J=7.70 \mathrm{~Hz}$, H-1'), 3.86 (br d, $J=11.00 \mathrm{~Hz}, \mathrm{H}-6$ 'b), 3.61 (dd, $J=11.00$, $6.00 \mathrm{~Hz}, \mathrm{H}-6$ 'a), 4.45 (d, $J=7.40 \mathrm{~Hz}, \mathrm{H}-1$ '), $3.23-3.60$ (8H, glycosyl protone). $-{ }^{13} \mathrm{C}$ NMR (100.6 $\left.\mathrm{MHz}, \mathrm{CD}_{3} \mathrm{OD}\right)$ : $\delta=39.91$ (C-1), 27.10 (C-2), 91.46 (C-3), 40.45 (C-4), 57.14 (C-5), 19.45 (C-6), 34.38 (C-7), 40.43 (C-8), 48.11 (C-9), 37.87 (C-10), 24.59 (C-11), 122.60 (C-12), 146.40 (C-13), 41.72 (C-14), 28.61 (C-15), 25.75 (C-16), 48.78 (C-17), 43.35 (C-18), 43.55 (C-19), 37.09 (C-20), 76.32 (C-21), 41.19 (C-22), 28.50 (C-23), 16.96 (C-24), 16.07 (C-25), 18.19 (C-26), 25.90 (C-27), (C-28 not observed), 27.92 (C-29), 25.76 (C-30), 104.67 (C-1'), 81.33 (C-2'), 77.84 (C-3'), 71.89 (C-4'), 78.25 (C-5'), 63.06 (C-6'), 105.38 (C-1"), 75.80 (C-2"), 78.25 (C-3"), 205.32 (C-4”), 73.54 (C-5"). - FAB-MS (negative): $m / z=763[\mathrm{M}-\mathrm{H}]^{-}$, 633 [(M-H)-130] $]^{-}, 471$ [(M-H)-130-162 $]^{-}$; FAB-MS (positive): $m / z=809[(\mathrm{M}-2 \mathrm{H}+2 \mathrm{Na})+\mathrm{H}]^{+} ; m / z=764[\mathrm{M}]^{+}$; HR-ESI MS (negative ion mode) $m / z=763.4328[\mathrm{M}-\mathrm{H}]^{-}$ (calcd. for $\mathrm{C}_{41} \mathrm{H}_{64} \mathrm{O}_{13}, 763.4323$ ).

$21 \alpha$-hydroxy olean-12-ene-28-oic acid 3 $\beta$-4-oxo-pentopyranoside (2): Amorphous powder, m. p. $232-235{ }^{\circ} \mathrm{C}$. $[\alpha]_{\mathrm{D}}^{20}=+3.2^{\circ}(\mathrm{MeOH}, c 0.25) .-{ }^{1} \mathrm{H} \mathrm{NMR}(400 \mathrm{MHz}$, $\left.\mathrm{CD}_{3} \mathrm{OD}\right): \delta=0.85,0.91,0.92,0.95,0.98,1.05,1.16(3 \mathrm{H}$ each, tertiary methyls), 0.80 (br d, $J=11.20 \mathrm{~Hz}$ ), 3.20 (dd, $J=11.70,4.30 \mathrm{~Hz}, \mathrm{H}-3 \alpha$ ), 3.42 (overlap. H-21 $\beta$ ), 5.24 (m, H-12), 4.34 (d, $\left.J=7.70 \mathrm{~Hz}, \mathrm{H}-1^{\prime}\right), 3.24$ (dd, $J=8.80$, $7.70 \mathrm{~Hz}, \mathrm{H}-2$ '), 3.39 (d, $J=8.80 \mathrm{~Hz}, \mathrm{H}-3^{\prime}$ ), 3.41 (d, $J=$ $9.40 \mathrm{~Hz}, \mathrm{H}-5$ 'a), 3.56 (d, $J=9.40 \mathrm{~Hz}, \mathrm{H}-5$ ' b).$-{ }^{13} \mathrm{C} \mathrm{NMR}$ (100.6 MHz, CD $\left.{ }_{3} \mathrm{OD}\right): \delta=39.93(\mathrm{C}-1), 27.85$ (C-2), 90.96 (C-3), 40.49 (C-4), 57.18 (C-5), 19.42 (C-6), 34.38 (C-7), 40.23 (C-8), 48.37 (C-9), 37.98 (C-10), 24.62 (C-11), 122.70 (C-12), 145.68 (C-13), 41.25 (C-14), 29.63 (C-15), 25.00 (C-16), 48.60 (C-17), 43.34 (C-18), 43.04 (C-19), 37.14 (C-20), 75.73 (C-21), 41.17 (C-22), 28.61 (C-23), 17.08 
(C-24), 16.11 (C-25), 18.20 (C-26), 25.87 (C-27), 180.46 (C-28), 27.98 (C-29), 25.82 (C-30), 106.70 (C-1'), 75.51 (C-2'), 78.08 (C-3'), 208.31 (C-4'), 73.72 (C-5'). - FABMS (negative): $m / z=601[\mathrm{M}-\mathrm{H}]^{-}, 471[(\mathrm{M}-\mathrm{H})-130]^{-}, 623$ $[(\mathrm{M}-\mathrm{H}+\mathrm{Na})-\mathrm{H}]^{-}, 645[(\mathrm{M}-2 \mathrm{H}+2 \mathrm{Na})+\mathrm{H}]^{-}, \mathrm{FAB}-\mathrm{MS}$ (positive $): m / z=647[(\mathrm{M}-2 \mathrm{H}+2 \mathrm{Na})+\mathrm{H}]^{+} ; m / z=602[\mathrm{M}]^{+}$; HR-ESI MS (negative ion mode) $\mathrm{m} / z=601.3798[\mathrm{M}-\mathrm{H}]^{-}$ (calcd. for $\mathrm{C}_{35} \mathrm{H}_{53} \mathrm{O}_{8}, 601.3795$ ).

Acid hydrolysis: Each glycoside $(5 \mathrm{mg})$ was refluxed with $7 \%$ methanolic $\mathrm{HCl}(5 \mathrm{ml})$ for $3 \mathrm{~h}$. The $\mathrm{MeOH}$ was evaporated, the mixture was diluted with $\mathrm{H}_{2} \mathrm{O}$, and the hydrolysate was partitioned between EtOAc and $\mathrm{H}_{2} \mathrm{O}$. The aglyconecontaining organic phase was concentrated, and analysed by TLC. The aglycone of $\mathbf{1}$ and $\mathbf{2}$ found to be identical with sapogenin 1a. The aqueous layer was neutralized with $\mathrm{Ag}_{2} \mathrm{CO}_{3}$, filtered, concentrated, and tested for carbohydrates by co-TLC with authentic samples using EtOAc$\mathrm{MeOH}-\mathrm{HAc}-\mathrm{H}_{2} \mathrm{O}(12: 3: 3: 2)$ as eluent. In addition the filtrate from 2 was evaporated to afford a residue, which was chromatographed on a silica gel column $(20 \mathrm{~g})$ with $\mathrm{CHCl}_{3}$ -
$\mathrm{MeOH}(1: 0,21 ; 95: 5,51)$ as eluent to give 4-oxo-pentose (ca. $1.2 \mathrm{mg}$ ). Positive FAB-MS $m / z: 131\left[(\mathrm{M}+\mathrm{H})-\mathrm{H}_{2} \mathrm{O}\right]^{+}$, $172[\mathrm{M}+\mathrm{H}+\mathrm{Na}]^{+}$.

Enzymatic hydrolysis: The glycosides (1 and $\mathbf{2}$ respectively, $2 \mathrm{mg}$ ) were dissolved in $5 \mathrm{ml}$ of $\mathrm{H}_{2} \mathrm{O}$ at $40{ }^{\circ} \mathrm{C}$. After cooling to r. t. $\mathrm{CH}_{3} \mathrm{COOH}(10 \%)$ was added to $\mathrm{pH}$ 5.5. To this mixture $2 \mathrm{mg}$ of Luizym ${ }^{\circledR}$, an enzyme preparation from Aspergillus oryzae (Luitpold Pharma, D-81379 München) was appended. After $5 \mathrm{~d}$ at r.t. in the dark the sediment was removed by filtration, the liquid was concentrated to $50 \mathrm{ml}$ under reduced pressure, and subsequently extracted three times with $50 \mathrm{ml}$ of $\mathrm{CHCl}_{3}$.

\section{Acknowledgements}

This work was supported by a grant 59/2003 from Medical Science Council (M. S. C.) at Medical University of Sofia. We are grateful to Dr R. Buyukliev (Sofia, Bulgaria) for helpful discussions.

The authors thank Mrs. H. Hillmann, Firma Schering AG, Berlin, Germany, for providing the HR-ESI MS spectra.
[1] P. A. Elenga, S. Nikolov, D. Panova, Pharmazie 42, 422 (1987).

[2] St. Nikolov, N. Benbassat, Pharmacia 44, 34 (1997).

[3] A. S. Gromova, V. I. Lutsky, J. G. Cannon, D. Li, N. L. Owen, Russian Chemical Bulletin, Int. Ed. 50, 1107 (2001).

[4] L. Verotta, M. Guerrini, N. A. El-Sebakhy, A. M. Assad, S. M. Toaima, M. M. Radwan, Y. D. Luo, J. M. Pezzuto, Planta Medica 68, 986 (2002).
[5] I. N. Krasteva, R. A. Toshkova, S. D. Nikolov, Phytother. Res. 18, 255 (2004).

[6] A. Inada, M. Yamada, H. Murata, M. Kobayashi, H. Toya, Y. Kato, T. Nakanishi, Chem. Pharm. Bull. 36, 4269 (1988).

[7] K. Ohtani, K. Ogawa, R. Kasai, C.R. Yang, K. Yamasaki, J. Zhou, O. Tanaka, Phytochemistry 31, 1747 (1992). 